\title{
Phenotypic Detection of Metallo $\beta$-Lactamase in Carbapenem Resistant Gram Negative Bacilli Isolates
}

\author{
SHAABAN R. HELAL, M.D.*; HEBAT-ALLAH G. RASHED, M.D.*; TAGHREED M. KAMAL EL-DIN, M.D.*; \\ MOHAMMED Z. ABD EL-RAHMAN, M.D.*; EBTISAM S. HASSANIN, M.Sc.*,****** and \\ SUGANO S., M.D.** \\ The Department of Clinical Pathology, Faculty of Medicine, Assiut University, Assiut, Egypt*, \\ The Department of Medical Genomics, Tokyo University, Minato-Ku, Tokyo, Japan** and \\ Assiut General Hospital, Ministry of Health, Assiut, Egypt***
}

\begin{abstract}
Background: A rising threat of the rapid spread of acquired metallo (3-lactamases (MBLs) among Gram Negative Bacilli $(\mathrm{GNB})$ is a matter of public health concern worldwide. Hence, detection of MBLs producing clinical isolates via an accurate and cost effective technique is necessary to prevent their dissemination as well as regulation of antimicrobial stewardship policy. This study aimed to detect MBLs in Carbapenem Resistant Gram Negative Bacilli (CRGNB) using two phenotypic tests; Combined Disc Test (CDT) and Double Disc Synergy Test (DDST).

Methods: We conducted a prospective study on $130 \mathrm{GNB}$ isolates recovered from different clinical specimens collected from different patients. Isolates were identified then antibiotic susceptibility profile was determined by VITEK $\AA 2$ compact system. GNB which showed resistance to meropenem were screened for MBLs production by CDT and DDST.
\end{abstract}

Results: Out of 36 CRGNB isolates, 23 isolates $(63.9 \%)$ were MBLs positive by CDT, and $20(55.6 \%)$ by DDST. With almost perfect agreement between CDT and DDST.

Conclusion: Our study validates two phenotypic methods (CDT and DDST) for the detection of MBLs production, making them highly applicable to routine clinical laboratories.

Key Words: MBLs - Combined disc test - Double disc synergy test $-C R G N B$

\section{Introduction}

RESISTANCE of pathogenic organisms to antibiotics is a worldwide problem with serious consequences on the treatment of infectious diseases. The increased use or misuse of antibiotics are major contributing factors that have resulted in economic losses to the consumer, hospital and society as more expensive drugs are used as secondline treatment, more tests need to be carried out

Correspondence to: Dr. Ebtisam S. Hassanin, E-Mail: roofy2006@yahoo.com and invariably the patient has to stay in the hospital for longer duration [1].

The introduction of carbapenems into clinical practice represented a great advance in the treatment of serious bacterial infections caused by 13 -lactam resistant bacteria, due to their broad spectrum of activity and stability to hydrolysis by most $\beta$ lactamases [2]. Carbapenems have been used as the last resort of antimicrobials in the treatment of serious infections caused by GNB. However, the clinical use of carbapenem is in danger with the emergence of carbapenem resistance, which become a public health problem around the world in terms of increased mortality, longer hospital stays, and higher costs [3]. Resistance to carbapenem is due to decreased outer membrane permeability, increased efflux systems, alteration of penicillinbinding proteins and carbapenem hydrolyzing enzymes (carbapenemase) which is the most widespread cause of carbapenem resistance [4]

Carbapenemases can be divided into metallocarbapenemases (zinc-dependent class B) and non metallo-carbapenemases (zinc-independent classes A and D) [5]. The MBLs was first detected in 1960, in Bacillus cereus which was chromosomal in location. Then, first plasmid mediated MBLs isolates was found in Pseudomonas aeruginosa in 1991 in Japan. Since early 1990s, MBLs encoding genes have been reported all over the world in clinically important pathogens, such as Pseudomonas spp., Acinetobacter spp., and members of the Enterobacteriaceae family [6]

Acquired MBLs is encoded by integron borne mobile gene cassettes, hence MBLs producing strains are often resistant to different classes of 
antimicrobial agents with transferable properties to various types of bacteria [7]. Thus, the detection of MBLs producing GNB is necessary to aid in appropriate treatment and infection control measures, and to prevent their dissemination [8] .

Detection of genes coding for MBLs usually gives reliable and satisfactory results. However, because of the cost of that method, it is of limited practical use for routine diagnostic microbiology laboratories. Thus, a simple and inexpensive phenotypic testing methods are more practical for detection of MBLs producers [9]. Several phenotypic methods have been described for detection of MBLs among clinical isolates as DDST, CDT and the MBLs Epsilometer test (E-test) [10] .

Microbiology laboratories must be prepared to screen for MBLs-producing isolates by a low cost, convenient and sensitive procedure. The present study was undertaken to detect MBLs in GNB using two phenotypic methods (CDT and DDST).

\section{Material and Methods}

This prospective study was conducted over a period of 8 months duration from October 2013 to June 2014 on 130 specimens ( 88 males and 42 females) of patients admitted to different wards/ ICUs at Assiut University Hospitals including Chest, Emergency, General ICU, Neurology, Burn, ENT, Tropical Medicine, Internal Medicine, Pediatrics, Coronary, Gynecology and Surgery Units. Samples include 34 endotracheal aspirates, 31 sputum samples, 22 wound swabs, 18 blood samples, 15 throat swabs, 6 urine samples, 3 pleural fluid samples and one vaginal swab. Collection of samples were done under strict aseptic precautions according to standard protocols and processed at once [11]

\section{Laboratory processing of the samples:}

Samples were processed both at Assiut University Hospitals, Clinical Pathology Department, Microbiology Unit, Egypt and at Tokyo University, Institute of Medical Science, Faculty of Forntier Sciences, Department of Medical Genomics, Prof. Sumio Sugano laboratory, Japan.

All samples were cultured directly on MacConkey agar (BD BactoTM, France). Blood samples were inoculated into BACT/ALERT® PF culture bottles, then incubated in the BACT/ALERT 3D system (BioMe'rieux, USA) for periodic reading. Subcultures on MacConkey agar were done for all BACTEC culture bottles with positive growth.
GNB were identified by Gram staining, colony morphology, biochemical reactions, API RapiD 20ETM (Sysmex BioMérieux, Japan) and VITEK ${ }^{\circledR}$ 2 Compact (BioMérieux, France). Then identification of all samples was confirmed with 16S rRNA gene sequencing, where Bacterial DNA was extracted using Gentra ${ }^{\circledR}$ Puregene ${ }^{\circledR}$ kit (a product of Qiagen ${ }^{\circledR}$ USA). We used universal primers fD 1 (5' AGAGTTTGATCCTGGCTCAG 3') and rP2 (5' ACGGCTACCTTGTTACGACTT 3') as general primers for $16 \mathrm{~S}$ ribosomal subunit amplification; supplied by Invitrogen ${ }^{\circledR}$, Life Technologies ${ }^{\circledR}$. The expected amplicon size was $1550 \mathrm{bp}$. PCR reactions were done using Thermo Scientific Phusion HighFidelity PCR Master Mix (ref F-531L) according to product manual. Then agarose gel electrophoresis was done and correct size PCR product was extracted from agarose gel using QIAquick ${ }^{\circledR}$ gel extraction kit. Sequencing was done using Sanger method. The BigDye ${ }^{\circledR}$ Terminator v3.1 Cycle Sequencing Kit was used. Sequences obtained were merged using online service by EMBOSS merger (http://emboss. bioinformatics.nl/cgi-bin/emboss/ merger) and then blasted using NCBI Blastn engine. Organisms with highest similarity was recorded.

\section{Antimicrobial susceptibility testing:}

VITEK® 2 compact system (AST-GNB card) (BioMe'rieux, France) was used for the automated quantitative susceptibility testing of isolated colonies for most clinically significant GNB. The following antimicrobials were included: Ampicillin, Ampicillin/Sulbactam, Piperacillin/Tazobactam, Cefazolin, Cefoxitin, Ceftazidime, Ceftriaxone, Cefepime, Meropenem, Amikacin, Gentamicin, Tobramycin, Ciprofloxacin, Levofloxacin, Nitrofurantoin and Trimethoprim/Sulfamethoxazole.

\section{Phenotypic detection of MBLs in CRGNB isolates:}

\section{A- Combined Disc Test (CDT):}

The inoculum was adjusted to a $0.5 \mathrm{McF}$ arland standard, and a Mueller-Hinton Agar plate (Oxoid Limited, England) was inoculated as recommended by CLSI for antibiotic sensitivity testing by the disc diffusion method. The presence of MBLs was determined by placing two 10 n meropenem discs (Oxoid, England) on the inoculated plate, in which 10 M EDTA was added to one of the meropenem discs. After overnight incubation at $37^{\circ} \mathrm{C}$, the inhibition zones of meropenem and meropenem-EDTA discs were compared. A zone difference of over $7 \mathrm{~mm}$ between the meropenem and the meropenem-EDTA inhibition zones confirmed the isolate to be MBLs-positive [12] 


\section{B- Double Disc Synergy Test (DDST):}

The inoculum was adjusted to a $0.5 \mathrm{McF}$ arland standard, and a Mueller-Hinton Agar plate was inoculated. One blank filter paper disc was treated with 10 M EDTA and placed on the center of inoculated plate. $10 \mu \mathrm{g}$ meropenem disc (Oxoid, England) was placed $20 \mathrm{~mm}$ away from the EDTA disc (measured center-to-center). After overnight incubation at $37^{\circ} \mathrm{C}$, Enhancement of inhibition zone in the area between the EDTA disc and MER disc in comparison with inhibition zone on the far side of the drug was interpreted as a positive result [13].

\section{Results}

Out of 130 GNB studied, 40 were Pseudomonas aeurogenosa strains, 27 Proteus mirabilis, 19 Providencia vermicola, 10 Serratia marcescens, 9 Providencia rettgeri, 8 Escherichia coli, 6 Providencia stuartii, 3 Citrobacter koseri, one Acinetobacter baumannii, one Alkaligenes faecalis, one Enterobacter cloacae, one Escherichia fergusonii, one Proteus penneri, one Pseudomonas putida, one Salmonella enterica and one Serratia species.

The resistance rates of GNB were found as $89.2 \%$ (no. 116) for ampicillin, $54.6 \%$ (no. 71 ) for ampicillin/sulbactam, $32.3 \%$ (no. 42) for piperacillin/tazobactam, $90.8 \%$ (no. 118) for cefazoline, $52.3 \%$ (no. 68) for cefoxitin, $43.8 \%$ (no. 57 ) for ceftazidime, $55.4 \%$ (no. 72 ) for ceftriaxone, $33.8 \%$ (no. 44) for cefepime, $20.8 \%$ (no. 27) for meropenem, $13.1 \%$ (no. 17) for amikacin, $19.2 \%$ (no. 25) for gentamicin, 27.7 (no. 36) for tobramycin, $58.5 \%$ (no. 76) for ciprofloxacin, 72.3\% (no. 94) for levofloxacin, $85.4 \%$ (no. 111) for nitrofurantoin and $66.2 \%$ (no. 86) for trimethoprim/ sulphamethoxazole (Table 1) \& Fig. (1).

Among 36 (27.7\%) meropenem resistant isolates, $22(61.1 \%)$ isolates were Pseudomonas spp., 6 (16.7\%) were Providencia spp., 3 (8.3\%) were E. coli spp., 2 (5.6\%) were Proteus spp., one (2.8\%) was Acinetobacter spp., one (2.8\%) was Enterobacter spp. and one (2.8\%) was Serratia spp.

The carbapenem resistant isolates were more frequently isolated from sputum samples 14 (3 8.9\%), followed by endotracheal aspirates 7 (19.4\%), followed by wound swabs $6(16.7 \%)$, followed by urine samples $4(11.1 \%)$, then throat swabs and pleural fluid equally $2(5.6 \%)$ and one blood sample (2.8\%).

The highest frequency of CRGNB isolates was found in Chest Department represented 9 isolates
(25.1\%) from total number of CRGNB isolates, followed by both general ICU and Emergency Unit represented 7 (19.4\%), Neurology Department represented 4 (11.1\%), both Pediatric Department and Burn Unit which represented $3(8.3 \%)$ and then ENT, Internal Medicine and Tropical Medicine Departments, one sample (2.8\%) from each department of them.

Out of 36 CRGNB isolates, 23 isolates $(63.9 \%)$ were MBLs positive by CDT Fig. (2), and 20 (55.6\%) by DDST Fig. (3).

Kappa value of 0.83 ( $p<0.001)$, indicating almost perfect agreement between CDT and DDST [14] as shown in (Table 2) and Fig. (4).

Table (1): Antibiotic sensitivity testing results.

\begin{tabular}{|c|c|c|c|}
\hline Antimicrobial agent & $\begin{array}{c}\text { Intermediate } \\
\text { No. }(\%)\end{array}$ & $\begin{array}{l}\text { Resistant } \\
\text { No. }(\%)\end{array}$ & $\begin{array}{c}\text { Susceptible } \\
\text { No. }(\%)\end{array}$ \\
\hline - Ampicillin & $0(0 \%)$ & $116(89.2 \%)$ & $14(10.8 \%)$ \\
\hline $\begin{array}{c}\text { - Ampicillin/ } \\
\text { Sulbactam }\end{array}$ & $0(0 \%)$ & $71(54.6 \%)$ & $59(45.4 \%)$ \\
\hline $\begin{array}{l}\text { - Piperacillin/ } \\
\text { Tazobactam }\end{array}$ & $5(3.8 \%)$ & $42(32.3 \%)$ & $83(63.8 \%)$ \\
\hline - Cefazoline & $0(0 \%)$ & $118(90.8 \%)$ & $12(9.2 \%)$ \\
\hline - Cefoxitin & $3(2.3 \%)$ & $68(52.3 \%)$ & $59(45.3 \%)$ \\
\hline - Ceftazidime & $0(0 \%)$ & $57(43.8 \%)$ & $73(56.2 \%)$ \\
\hline - Ceftriaxone & $1(0.8 \%)$ & $72(55.4 \%)$ & $57(43.8 \%)$ \\
\hline - Cefepime & $5(3.8 \%)$ & $44(33.8 \%)$ & $81(62.3 \%)$ \\
\hline - Meropenem & $9(6.9 \%)$ & $27(20.8 \%)$ & $94(72.3 \%)$ \\
\hline - Amikacin & $6(4.6 \%)$ & $17(13.1 \%)$ & $107(82.3 \%)$ \\
\hline - Gentamicin & $11(8.5 \%)$ & $25(19.2 \%)$ & $94(72.3 \%)$ \\
\hline - Tobramycin & $12(9.2 \%)$ & $36(27.7 \%)$ & $82(63.1 \%)$ \\
\hline - Ciprofloxacin & $13(10 \%)$ & $76(58.5 \%)$ & $41(31.5 \%)$ \\
\hline - Levofloxacin & $5(3.8 \%)$ & $94(72.3 \%)$ & $31(23.8 \%)$ \\
\hline - Nitrofurantoin & $1(0.8 \%)$ & $111(85.4 \%)$ & $18(13.8 \%)$ \\
\hline $\begin{array}{l}\text { - Trimetho/ } \\
\text { Sulphamethox } \\
\text { azole }\end{array}$ & $0(0 \%)$ & $86(66.2 \%)$ & $44(33.8 \%)$ \\
\hline
\end{tabular}

Table (2): Agreement between CDT and DDST ( $\mathrm{n}=36)$.

\begin{tabular}{|c|c|c|c|}
\hline & \multicolumn{2}{|c|}{ DDST } & \multirow{2}{*}{$p$-value } \\
\hline & Negative & Positive & \\
\hline \multicolumn{4}{|l|}{ CDT: } \\
\hline Negative & $13(36.1 \%)$ & $0 \quad(0 \%)$ & $<0.001 *$ \\
\hline Positive & $3 \quad(8.3 \%)$ & $20(55.6 \%)$ & \\
\hline Карра & \multicolumn{2}{|c|}{$83 \%$} & $<0.001$ \\
\hline
\end{tabular}

* Chi-square test was used to compare the difference in proportions between groups.

* \% calculated from total number. 


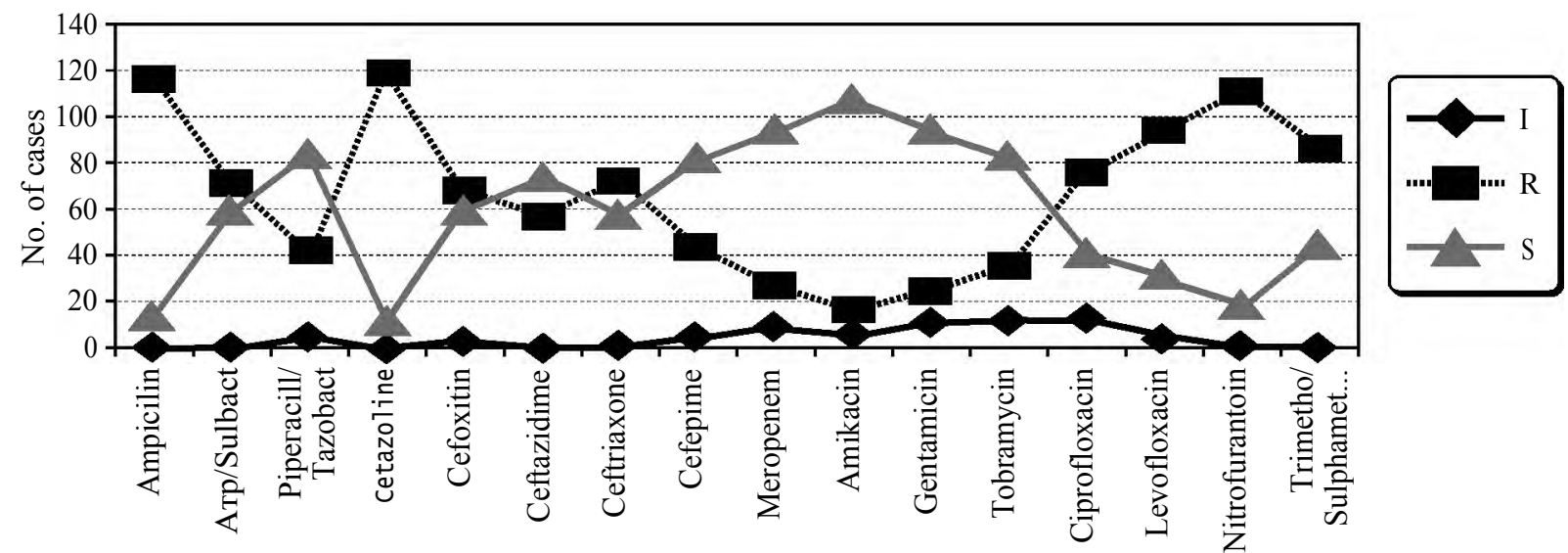

Fig. (1): Antibiotic sensitivity testing results.

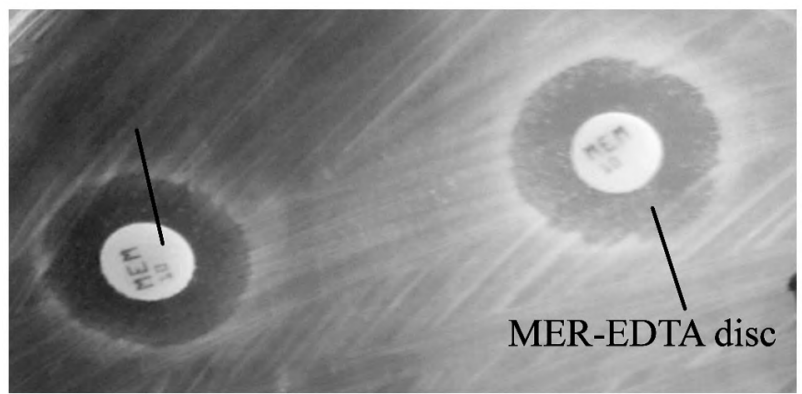

(A)

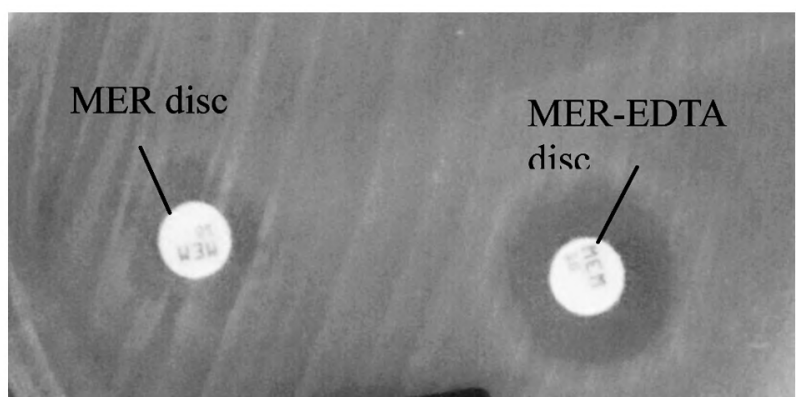

(B)

Fig. (2): CDT (A) Negative (B) Positive.

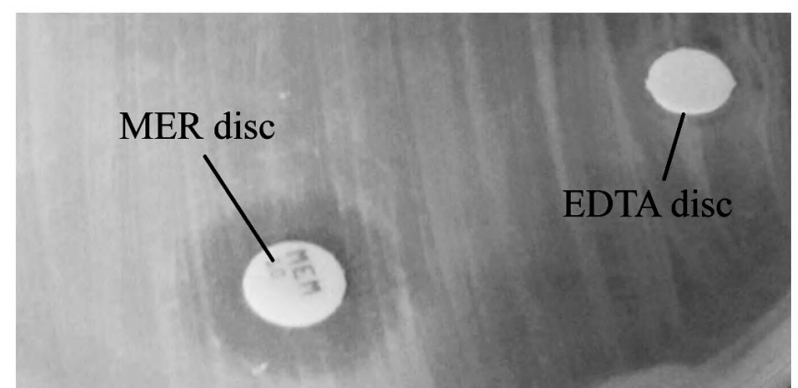

(A)

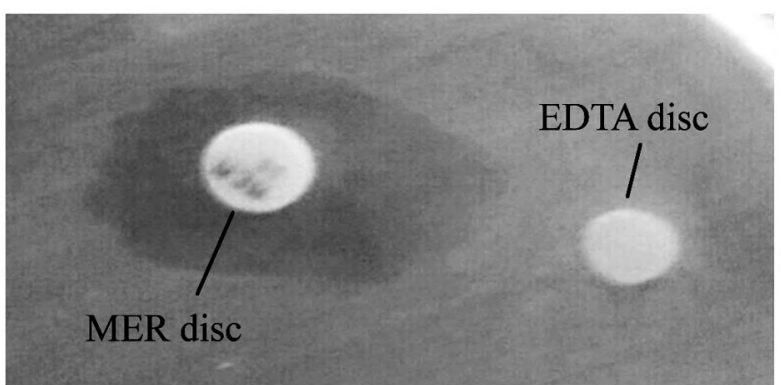

(B)

Fig. (3): DDST (A) Negative (B) Positive.

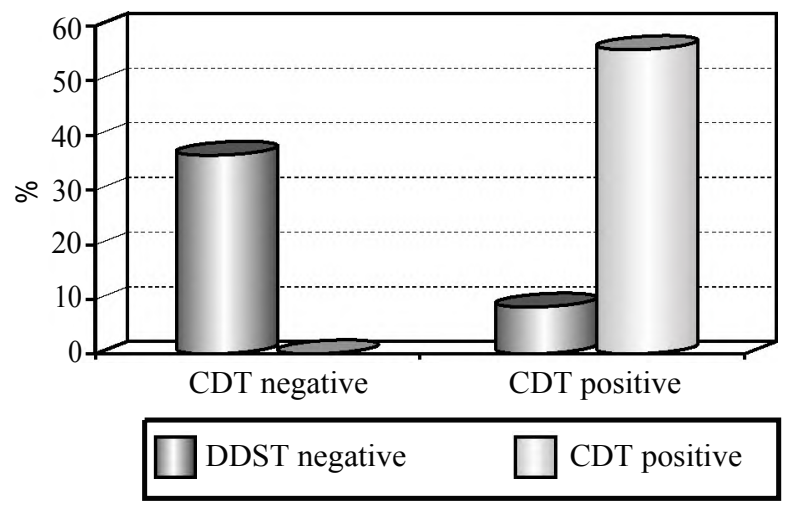

Fig. (4): Agreement between CDT and DDST.

\section{Discussion}

Recent events indicate an increasing occurrence of antimicrobial resistance in GNB. Progressive antimicrobial resistance to most classes of antibiotics, including carbapenems, has made treatment of infection caused by these bacteria particularly difficult, even with combination therapy [15]. The clinical impact of carbapenem resistance has become a public health problem around the world in terms of increased mortality, longer hospital stays, and higher costs [3]. In the Middle East, the occur- 
rence of CRGNB is increased, this increase could be due to extensive use of carbapenems which has likely generated a selective antibiotic pressure [16]

The isolated GNB in our study were most resistant to cefazoline (90.8\%), while amikacin was found to be the most sensitive drug. Piéboji et al., [17], Lockhart et al., [18], Sharif et al., [19], Panchal et al., [9] and Zaki et al., [10] reported that isolated GNB were most resistant to Amoxicillin, ampicillin-sulbactam, ampicillin, Tetracycline and gentamicin then trimethoprim respectively.

The carbapenem resistance rate among GNB in our study was $20.8 \%$. This resistance rate is in accordance with many other investigators, as Zaki et al., [10] reported that carbapenem resistance among GNB isolated from Mansoura University Hospital in Egypt was 28\%. Panchal et al., [9] reported $28 \%$ carbapenem resistance rate among GNB isolates in a rural hospital in India. Wattal et al., [20] reported high prevalence of resistance to carbapenems ranging from 13 to $51 \%$ in E. coli and Klebsiella spp. from ICUs and wards from a tertiary care hospital in Delhi. Gupta et al., [21] also reported high prevalence of resistance varying from 17 to $22 \%$ to various carbapenems among Enterobacteriaceae strains. While carbapenem resistance among NFGNB isolates according to Das et al., [22] and Rathod et al., [23] was $63 \%$ and $28 \%$, respectively. Misuse and abuse of antibiotics in Egypt has adversely contributed to the rate of antibiotic resistance in the study and might also be the cause of the emergence of carbapenemase enzymes. However, our results are different from some investigators, Navaneeth et al., [24] reported a prevalence of $12 \%$ carbapenem resistance among 50 strains of GNB isolated from various clinical specimens. Also, Mohammadzadeh et al., [2] found that carbapenem resistance rate in GNB isolates was $7 \%$, which means a low prevalence of carbapenem resistance in last two studies in comparison with our study. This difference may be attributed to different patient population with different underlying diseases, different environment and different numbers of investigated specimens.

In our study, the carbapenem resistant isolates were more frequently isolated from sputum samples $14(38.9 \%)$, followed by endotracheal aspirates 7 (19.4\%), followed by wound swabs $6(16.7 \%)$, followed by urine samples 4 (11.1\%), then throat swabs and pleural fluid equally $2(5.6 \%)$ and one blood sample (2.8\%). Among these; $22(61.1 \%)$ isolates were Pseudomonas spp., 6 (16.7\%) were Providencia spp., 3 (8.3\%) were E. coli spp., 2 (5.6\%) were Proteus spp., one (2.8\%) was Acineto- bacter spp., one (2.8\%) was Enterobacter spp. and one $(2.8 \%)$ was Serratia spp., Omair et al., (2012) found that out of 52 CRGNB, 23 (44\%) were from nasobronchial lavage, 10 (19\%) were from pus, 6 (11\%) from urine, 5 (10\%) from catheter tips, 3 (6\%) from blood, 2 (4\%) from sputum, 2 (4\%) from ear swabs and only one ( $2 \%)$ from high vaginal swab. Among these 52 isolates, 33 (63\%) were Acinetobacter spp., 10 (19.5\%) were Pseudomonas spp. and $9(17.5 \%)$ were Escherichia coli., and Mohammadzadeh et al., [2] reported that among 62 imipenem resistant isolates 35 (56.5\%), $24(38.7 \%)$ and $3(4.9 \%)$ isolates were Acinetobacter spp., Pseudomonas spp. and Enterobacteriaceae respectively, where $50(80 \%)$ were sputum samples, 12 (19\%) were blood samples, $4(0.4 \%)$ were wound samples and $6(0.9 \%)$ were other samples. While in a study by Diwakar et al., [25], 30 out of 110 CRGNB strains were Klebsiella pneumonia strains, 26 Escherichia coli strains, 15 Citrobacter freundii, 13 Pseudomonas aeruginosa strains, 13 Acinetobacter Baumanni strains, 5 Citrobacter koseri strains, 4 Enterobacter spp. Strains, 3 strains of Proteus mirabilis and 2 strains of Proteus vulgaris isolated. Maximum number of sample was urine 29 (26.4\%) followed by pus 27 (24.5\%), blood $22(20 \%)$ and $32(29.1 \%)$ from other infections.

Resistance to carbapenems is often mediated by production of carbapenem hydrolyzing enzymes; carbapenemases which have been reported in several countries and have emerged as the most important mechanism of carbapenem resistance. carbapenemases can be divided into metallo-carbapenemases and non metallo-carbapenemases [5] Infections due to MBLs producing GNB are a cause of high mortality and morbidity. Early detection by an economic and accurate method may aid in appropriate treatment and infection control measures [26].

The emergence of MBLs producing GNB is challenge to microbiology laboratories because there are no standardized guidelines available to detect them. PCR usually gives reliable and satisfactory results; however, because of the cost of that method, it is of limited practical use for routine diagnostic microbiology laboratories. Thus, a simple and inexpensive testing method for detection of MBLs producers is necessary [9].

The MBLs-producing GNB have now been reported in many geographic regions. In our study, prevalence of MBLs production in GNB was $63.9 \%$ using CDT and 55.6\% using DDST. Similar study conducted by Das et al., [20] showed that $67 \%$ of 
isolates were MBLs positive by CDT and 58\% were positive by DDST. The results of present study also correlate with the results of study conducted by Panchal et al., [9] in which $63 \%$ of the carbapenem resistant strains were MBLs producers by CDT and $53 \%$ by DDST. Diwakar et al., [23] also reported that $47 \%$ of GNB isolates were MBLs producers by CDT. While by using DDST Zaki et al., [10] found that $46.8 \%$ of isolates were MBLs positive. Our results were lower than those obtained by Pandya et al., [6] who found that $96.3 \%$ of isolates were MBLs positive by CDT and $81.5 \%$ were positive by DDST. Galani et al., [27] also reported that $94.7 \%$ of the carbapenem resistant strains were MBLs producers by CDT and $100 \%$ by DDST. Similar results were obtained by the study conducted by Picao et al., [28] in which $80 \%$ of isolates were identified as MBL producers by CDT and $82 \%$ by DDST. To the contrary, Thapa et al., [29] reported that MBLs production among GNB was 5.8\%. Also, Mishra [30] found that 1.3\% of the isolates were MBLs positive. This difference may be attributed to different patient population and different environment. In the present study there is almost perfect agreement between CDT and DDST.

\section{Conclusion:}

Our study validates two phenotypic methods (CDT and DDST) for the detection of MBLs production. Phenotypic methods are simple to perform, and the materials used are cheap, nontoxic, and easily accessible, making them highly applicable to routine clinical laboratories. Genetic confirmation by PCR may be used for positive isolates screened by phenotypic tests.

Financial support: No financial support. interest.

Conflicts of interest: There are no conflicts of

\section{Acknowledgment:}

Special thanks to Faculty of Medicine-Assiut University and Faculty of Forntier Sciences, Institute of Medical Science-Tokyo University, for providing us with all kinds of facilities.

\section{References}

1- SHAGUFTA R., FOMDA B.A., GULNAZ B., LUBNA S., JAN A., MOHD S. and JUNAID A.: Prevalence of AmpC Beta-lactamase in Gram Negative Bacilli by Different Phenotypic Methods in a Tertiary Care Institute in Kashmir. B.J.M.M.R., 19 (2): 1-9, 2017.

2- MOHAMMADZADEH M., TAVAKOLI M., MOHEBI A. and AGHAYI S.: Phenotypic and Genotypic Detection of Metallo-Beta-Lactamases among Imipenem Resistant
Gram Negative Isolates. J. Med. Bacterial., 5 (3): 36-42, 2016.

3- VANEGAS J.M., PARRA O.L. and JIMÉNEZ J.N.: Molecular epidemiology of carbapenem resistant gramnegative bacilli from infected pediatric population in tertiary care hospitals in Medellín, Colombia: An increasing problem. BMC Infectious Diseases, 16: 463-73, 2016.

4- SEVITHA B., RENU S. and ZENITH E.: Carbapenem resistance in clinically significant non fermenting gram negative bacelli. Journal of Evolution of Medical and Dental Sciences, 2 (47): 9131-4, 2013.

5- JEON J.H., LEE J.H., PARK K.S., LEE J.J., KARIM A.M., LEE C., JEONG B.C. and LEE S.H.: Structural Basis for Carbapenem-Hydrolyzing Mechanisms of Carbapenemases Conferring Antibiotic Resistance. Int. J. Mol. Sci., 16: 9654-92, 2015.

6- PANDYA P.N., PRAJAPATI B.S., MEHTA J.S., KIKANI M.K. and JOSHI J.P.: "Evaluation of various methods for detection of MBL (MBL) production in Gram negative bacilli," International Journal of Biological and Medical Research, 2 (3): 775-7, 2011.

7- VALENZA G., JOSEPH B., ELIAS J., CLAUS H., OETTERLEIN A., ENGELHARDT K., TURNWALD D., FROSCH M., ABELE-HORN M. and SCHOEN C.: First survey of Metallo 3 -lactamases in clinical isolates of Pseudomonas aeruginosa in a German University hospital. Antimicrob. Agents Chemother., 54: 3493-7, 2010.

8- CORNAGLIA G., GIAMARELLOU H. and ROSSOLINI G.M.: Metallo $\beta$-lactamases: A last frontier for $\beta$-lactams? Lancet Infec. Dis., 11: 381-93, 2011.

9- PANCHAL C.A., OZA S.S. and MEHTA S.J.: Comparison of four phenotypic methods for detection of metallo- Plactamase-producing Gram-negative bacteria in rural teaching hospital. J. Lab. Physicians., 9: 81-3, 2017.

10- ZAKI M.E., MASHALY M. and EL-AGAMY R.: Phenotypic Detection and Genotypic Characterization of MetalloBeta-Lactamases in Gram Negative Bacilli isolated from Mansoura University Hospital in Egypt. Int. J. Curr. Microbiol. App. Sci., 6 (2): 1496-505, 2017.

11- COLLEE J.K., MILES R.S. and WAN B.: Tests for the identification of bacteria. In: Mackie and McCartney Practical Medical Microbiology, 14th ed. 131-50, 1996.

12- YONG D., LEE K., YUM J.H., SHIN H.B., ROSSOLINI G.M. and CHONG Y.: Imipenem-EDTA disk method for differentiation of MBL producing clinical isolates of Pseudomonas spp. and Acinetobacter spp. J. Clin. Microbiol., 40: 3798-801, 2002.

13- LEE K., LIM Y.S., YONG D., YUM J.H. and CHONG Y.: Evaluation of Hodge test and the imipenem-EDTA double disk synergy test for differentiating MßL producing isolates of Pseudomonas spp. And Acinetobacter spp. J. Clin. Microbiol., 41: 4623-9, 2003.

14- LANDIS J.R. and KOCH G.G.: The measurement of observer agreement for categorical data. Biometrics, 33 (1): 159-74, 1977.

15- KHUNTAYAPORN P., MONTAKANTIKUL P., MOOTSIKAPUN P., THAMLIKITKUL V. and CHOMNAWANG M.T.: "Prevalence and genotypic relatedness of carbapenem resistance among multidrugresistant P. Aer- 
uginosa in tertiary hospitals across Thailand". Annals of Clinical Microbiology and Antimicrobials, 11 (25): 118690, 2012.

16- MOHAMMED E.H., FAKHR A.E., MOHAMMED E.I., SAYED H.M., AL-JOHERY S.A. and ABDEL GHANI W.A.: Spread of TEM, VIM, SHV, and CTX-M BLactamases in Imipenem-Resistant Gram-Negative Bacilli Isolated from Egyptian Hospitals. Int. J. Microbiol., e8382605, 2016.

17- PIÉBOJIA J.G., KOULLA-SHIROB S., NGASSAMA P., ADIOGOB D., NJINEA T. and NDUMBE P.: Antimicrobial resistance of Gram negative bacilli isolates from inpatients and outpatients at Yaounde Central Hospital, Cameroon. International Journal of Infectious Diseases, 8: 147-54, 2004.

18- LOCKHART S.R., MURRAY A.A., SUSAN E.B., GALE G., RIEDEL S., QUINN J.P. and DOERN G.V.: Antimicrobial Resistance among Gram-Negative Bacilli Causing Infections in Intensive Care Unit Patients in the United States between 1993 and 2004. J. Clin. Microbiol., 10: 3352-9, 2007.

19- SHARIF A., SHARIF M.R. and ALIZARGAR J.: Antibiotic Resistance of Gram-Negative Bacteria to Ampicillin, Gentamicin and Imipenem in Samples of the Patients Admitted to Shahidbeheshti Hospital in Kashan. World Applied Sciences Journal, 30 (6): 778-81, 2014.

20- WATTAL C., GOEL N., OBEROI J.K., RAVEENDRAN R., DATTA S. and PRASAD K.J.: Surveillance of multidrug resistant organisms in a tertiary care hospital in Delhi, India. J. Assoc. Physicians India, 58: 32-6, 2010.

21- GUPTA E., MOHANTY S., SOOD S., DHAWAN B., DAS B.K. and KAPIL A.: Emerging resistance to carbapenems in a tertiary care hospital in North India. Indian. J. Med. Res., 124: 95-8, 2006.

22- DAS N.K., GROVER N., SRIRAM R., KUMAR M., DUDHAT V.L. and PRASANNA S.: Prevalence of Carbapenem Resistance and Comparison between Different Phenotypic Methods for Detection of Metallo-BLactamases in Gram Negative Non-Fermentative Bacteria in the Acute Wards of a Tertiary Care Centre. Int. J. Curr. Microbiol. App. Sci., 5 (5): 109-19, 2016.

23- RATHOD R., GUPTA P., VEGAD M., SONI S. and PADARIA N.: Prevalence of Metallo Beta-Lactamase in Clinical Isolates of Non-Fermenters in A Tertiary Care Hospital. Nat. J. Integr. Res. Med., 8 (2): 149-52, 2017.

24- NAVANEETH B.V., SRIDARAN D., SAHAY D. and BELWADI M.R.: A preliminary study on metallo-betalactamase producing Pseudomonas aeruginosa in hospitalized patients. Indian. J. Med. Res., 116: 264-7, 2010.

25- DIWAKAR J., VERMA R.K., SINGH D.P., SINGH A. and KUMARI S.: Phenotypic detection of carbapenem resistance in gram negative bacilli from various clinical specimens of a tertiary care hospital in Western Uttar Pradesh. Int. J. Res. Med. Sci., 5: 3511-4, 2017.

26- OMAIR M., USMAN J., KALEEM F., HASSAN A., KHALID A. and FAHIM Q.: Evaluation of combined disc method for the detection of metallo- 3 -lactamase producing Gram negative bacilli. Malaysian Journal of Microbiology, 8 (1): 21-5, 2012.

27- GALANI I., REKATSINA P.D., HATZAKI D., PLACHOURAS D., SOULI M. and GIAMARELLOU H.: Evaluation of different laboratory tests for the detection of metallo-ßlactamase production in Enterobacteriaceae. J. Antimicrob. Chemother., 61: 548-53, 2008.

28- PICAO R.C., ANDRADE S.S., NICOLETTI A.G., CAMPANA E.H., MORAES G.C. and MENDES R.E.: Metalloß-Lactamase Detection: Comparative Evaluation of Double Disk Synergy versus Combined Disk Tests for IMPGIM-, SIM-, SPM-, or VIM Producing Isolates. J. Clin. Microbiol., 46 (6): 2028-37, 2008.

29- THAPA P., BHANDARI D., SHRESTHA D., PARAJULI H., CHAUDHARY P., AMATYA J. and AMATYA R.: A hospital based surveillance of metallo-beta-lactamase producing gram negative bacteria in Nepal by imipenemEDTA disk method. B.M.C. Res. Notes, 10: 322-8, 2017.

30- MISHRA S.K.: Metallo-beta-lactamase producing gramnegative bacterial isolates. J. Nepal. Health Res. Counc., 10 (22): 208-13, 2012.

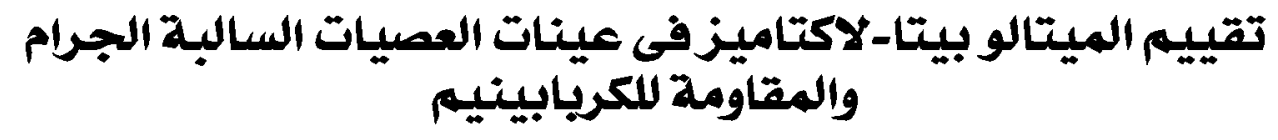

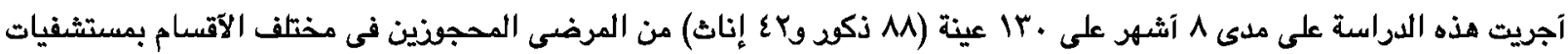

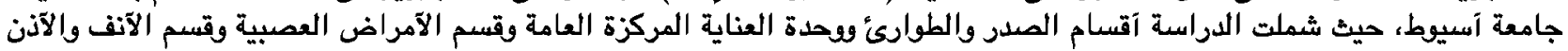

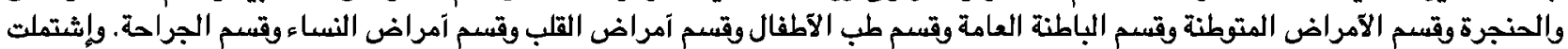

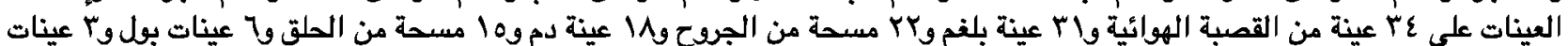

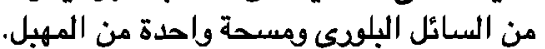

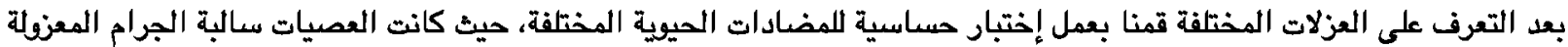

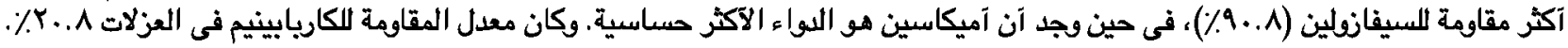

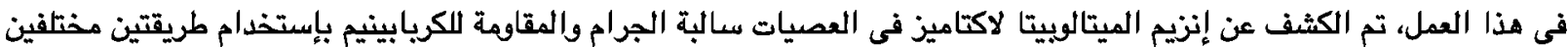

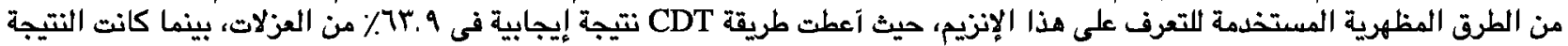
إيجابية في 7 \% \% من الحالات بإستخدام طريقة DDST. مع وجدي إتفاق مثالى تقريبا بين الإختبارين. 